Article

\title{
Focal plane array infrared imaging of linear polymeric patterns
}

\author{
Reo Honda ${ }^{1}$, Meguya Ryu ${ }^{1}$, Masayuki Moritake ${ }^{1}$, Armandas Balčytis ${ }^{2}$, Vygantas Mizeikis ${ }^{3}$, \\ Jitraporn Vongsvivut ${ }^{4}$, Mark J. Tobin ${ }^{4}$, Dominique Appadoo ${ }^{5}$, Jing-Liang $\mathrm{Li}^{6}$, Soon Hock $\mathrm{Ng}^{2}$, \\ Saulius Juodkazis ${ }^{2,7,8 *}$ and Junko Morikawa ${ }^{1 *}$ \\ 1 Tokyo Institute of Technology, Meguro-ku, Tokyo 152-8550, Japan \\ 2 Swinburne University of Technology, John Street, Hawthorn, Victoria 3122, Australia \\ 3 Research Institute of Electronics, Shizuoka University, Naka-ku, 3-5-3-1 Johoku, Hamamatsu, Shizuoka \\ 4328561, Japan \\ 4 Infrared Microspectroscopy Beamline, Australian Synchrotron, Clayton, Victoria 3168, Australia \\ $5 \mathrm{THz}$ - Far Infrared Beamline, Australian Synchrotron, Clayton, Victoria 3168, Australia \\ 6 Institute for Frontier Materials, Deakin University, Geelong, Victoria 3220, Australia \\ 7 Tokyo Tech World Research Hub Initiative (WRHI), School of Materials and Chemical Technology, Tokyo \\ Institute of Technology, 2-12-1, Ookayama, Meguro-ku, Tokyo 152-8550, Japan \\ 8 Melbourne Center for Nanofabrication, Australian National Fabrication Facility, Clayton, Victoria 3168, \\ Australia \\ * Correspondence: morikawa.j.aa@m.titech.ac.jp (JM); sjuodkazis@swin.edu.au (SJ)
}

\begin{abstract}
A focal plane array (FPA) detector was used for hyperspectral imaging in the infrared (IR) spectral region using thermal and synchrotron light sources. FPA Fourier-transform IR (FTIR) imaging microspectroscopy will be able to monitor real time changes at specific absorption bands when combined with high brightness synchrotron source. In this study, several types of samples with unique structural motifs were selected and used for assessing the capability of the FPA-FTIR imaging technique. It was shown that the time required for polariscopy at IR wavelengths can be substantially reduced by the FPA-FTIR imaging approach. By using natural and laser fabricated polymers with sub-wavelength features, alignment of absorbing molecular dipoles was revealed as well as higher order patterns (laser fabricated structures). Micro-spectroscopy of absorber orientation reveals alignment patterns even when they are not spatially resolved.
\end{abstract}

Keywords: focal plane array, thermal source, synchrotron radiation, infrared spectroscopy, hyperspectral imaging, silk

\section{Introduction}

For the spectral mapping of processes and material modifications in real time, data acquisition with a focal plane array (FPA) becomes a prerequisite in infrared (IR) microspectroscopy. This is particularly important for spectral characterisation of materials using Fourier transform IR (FTIR) microspectroscopic systems at synchrotron facilities where the extracted IR beam possesses the highest brightness. Polariscopy measurements to establish the polarisation state of light at long IR-THz spectral range is still at an early stage of development, mainly due to lack of optical elements, filters and waveplates [1]. This shortcoming can be easily overcome since fabrication resolution is not a limiting factor and there is a range of materials with high transmittance in IR, such as silicon (Si).

In this study, we tested the potential application of the FPA detector on FTIR microspectroscopic systems for hyperspectral imaging measurement using a thermal IR (Globar(TM)) source and synchrotron radiation [2]. Several types of samples with unique structural motifs were selected for the tests, including brown silk (representative of linear bio-polymer) [3-6], laser polymerised micro-optical element with a polymeric grating motif and as a reference sample a concentric grating in gold. It was found that imaging parameters (such as focal spot size and pixel size) played a critical 


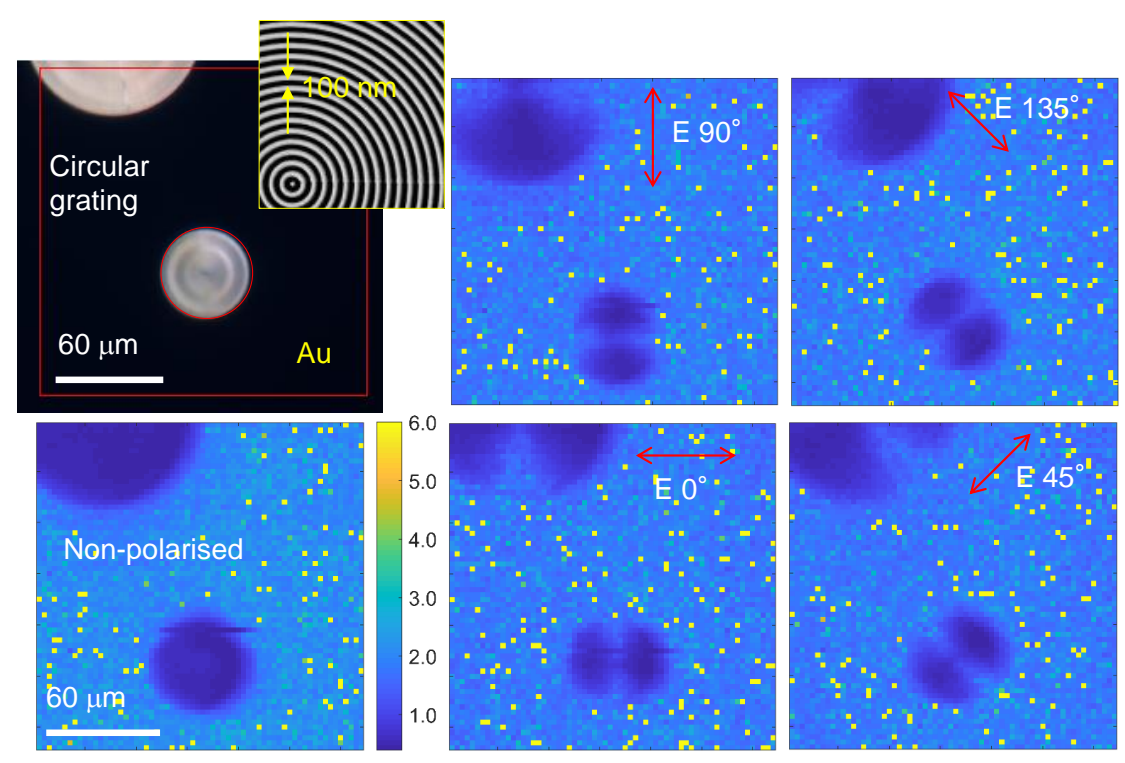

Figure 1. Single wavenumber $3000 \mathrm{~cm}^{-1}$ polarisation dependence of the absorbance $A=-\log (T)$, where $T$ is transmittance, measured through a circular grating; orienation of the E-field is marked. The light source was thermal-IR. FPA-FTIR images were taken using $64 \times 64$ pixels of the FPA detector. The pixel size was $2.8 \mu \mathrm{m}$, the Cassegrainian objective lens magnification was $15^{\times}$, spectral resolution $8 \mathrm{~cm}^{-1}$, accumulation over 128 scans. The grating was milled by $\mathrm{Ga}^{+}$-ions in a 100 -nm-thick $\mathrm{Au}$ sputtered on 30- $\mu \mathrm{m}$-thick sapphire; grating: 100-nm-half-pitch (see SEM image in the inset).

role in interpreting the FPA-FTIR images obtained from these sub-wavelength features. Based on the results presented here, the FPA-FTIR technique demonstrated its potential as a powerful analytical tool to underpin the research in the areas of the design of IR optical elements, and to be adopted for polariscopy measurements in IR and $\mathrm{THz}$ wavelengths.

\section{Results}

Three samples with sub-wavelength features, including (i) circular metal grating, (ii) laser polymerised radial structure, and (iii) natural brown silk, were used for FPA-FTIR microspectroscopic imaging measurements.

\subsection{Circular sub-wavelength grating}

The first FPA-FTIR experiments were carried out using a thermal IR (Globar(TM)) source. The reference sample with sub-wavelength circular grooves with the period of $200 \mathrm{~nm}$ and half-pitch spacing of $100 \mathrm{~nm}$ was used. The grating was milled with focused ion beam (FIB; IonLiNE Raith, Dortmund, Germany) through 100-nm-thick Au sputtered film, using a thin sapphire as a substrate (Fig. 1). Transmission $T$ at $3000 \mathrm{~cm}^{-1}$ wavenumber $(\lambda=3.3 \mu \mathrm{m})$ was collected at four polarisations of incident thermal radiation. A clear dipolar nature of transmission was observed through the concentric grating; the 100-nm-thick Au film blocked direct transmission. Light with E-field aligned with the local orientation of the wavevector of the grating $\mathbf{k}: \mathbf{E} \| \mathbf{k}$ has lower absorbance, while in the locations where $\mathbf{E} \perp \mathbf{k}$ a stronger absorbance (lower $T$ ) was observed yielding a dipole pattern. From the absorbance determined at four orientations of linearly polarised illumination, it is possible to determine the orientation of the absorbing species/structures [8]. Using this method, the orientation amplitude and azimuth were determined as summarised in Fig. 2. The orientation azimuth angle $\theta^{\prime}$ was measured from the fit taken at four $\theta$ angles where the orientation strength is defined by the amplitude of absorbance $A_{\max }-A_{\min }$ [7]. The strongest orientation amplitude was at the edge of the circular grating where the curvature of the pattern was the lowest and, in the center, a less defined 
(a)
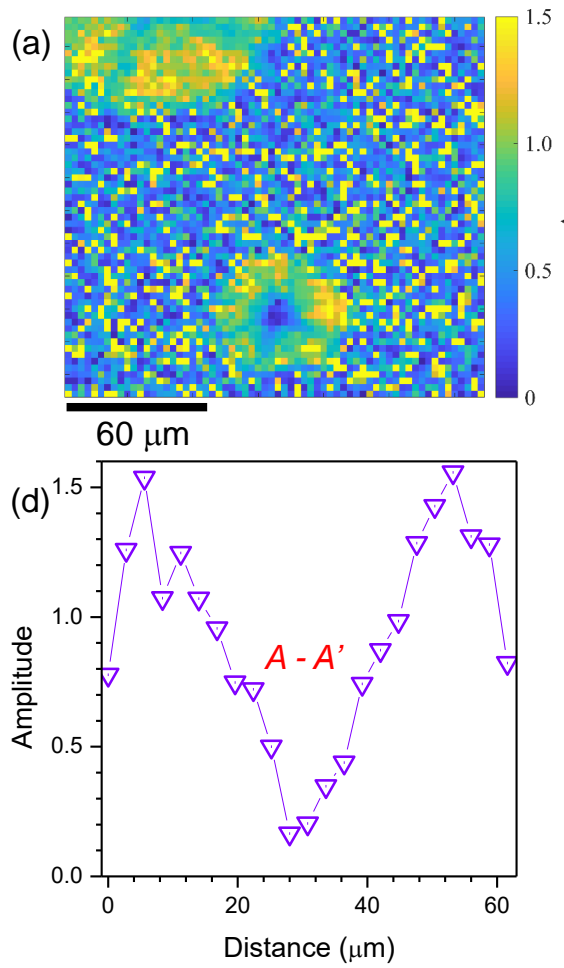

(b)

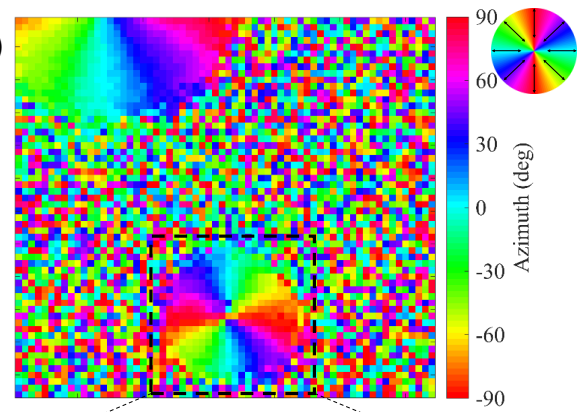

(c)

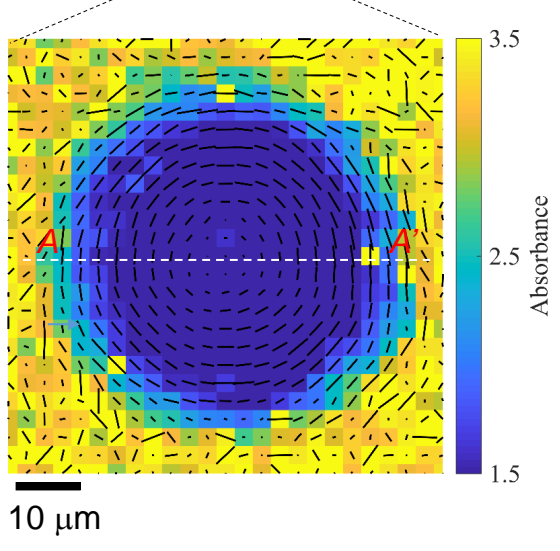

Figure 2. Circular grid reference measured with FPA; thermal-IR source. Orientation amplitude Amp (a), orientation azimuth (b), absorbance map overlaid with an orientation map (c), and central cross section $A-A^{\prime}(\mathrm{d})$ all at $3000 \mathrm{~cm}^{-1}$.

orientation amplitude was observed due to the strongest local curvature of the pattern (Fig. 2(a)). The azimuth (b) showed the expected circular geometry of the concentric grating as shown in (c) combined with absorbance plot; the cross section of absorbance amplitude is shown in (d).

\subsection{SZ2080 polymerised gratings}

Micro-optical diffractive elements made of gratings with an azimuthally changing orientation and period $\Lambda \approx 400 \mathrm{~nm}$ (duty cycle of 0.5 ) were polymerised by direct laser writing [9]. Such gratings with azimuthally varying optical axis orientation defined as $\psi=q \phi$, where $q$ is the half-integer and $\phi$ is the polar angle in cylindrical coordinates are called q-plates [10,11]; a usual grating is a $q=0$ plate while the concentric grating (Fig. 1(a)) is $q=1$. Q-plates are used as optical vortex generators [12] applicable to opto-mechanical manipulation of matter [13]. When the propagation phase reaches the $\lambda / 2$ condition the most efficient conversion of the incoming spin angular momentum (SAM) $\sigma$ into the orbital angular momentum (OAM) with a spiraling wavefront is achieved. The azimuthal phase $2 q \psi$ is defining the topological charge of the optical vortex $l=2 q \sigma$, where $\sigma= \pm 1$ is the spin for the left- or right-hand polarisation, respectively.

Q-plate $q=1$ was laser polymerised in SZ2080 resist with segments of $\Lambda=2.5 \mu \mathrm{m}$ period grating (Fig. 3(a)). The polymerised bars were approximately $1 \mu \mathrm{m}$ in width. For operation of q-plates at IR wavelengths, it is important to have a weak absorbance in the polymer and the height to satisfy a $\pi$ retardation for the propagating phase. Q-plates were polymerised on a 100-nm-thick $\mathrm{Si}_{3} \mathrm{~N}_{4}$ membrane. Point spectra from the q-plate and $\mathrm{Si}_{3} \mathrm{~N}_{4}$ membrane are shown in Fig. $3(\mathrm{~b})$. The orientational dependencies at four angles were measured (Fig. 3(c)) to determine the azimuthal distribution of the absorbers [8]. The $\mathrm{Si}_{3} \mathrm{~N}_{4}$ nano-membrane showed almost no absorption over the entire spectral window. Spectral features of SZ2080 at $2923 \mathrm{~cm}^{-1}$ due to C-H stretching in alkanes 
(a)

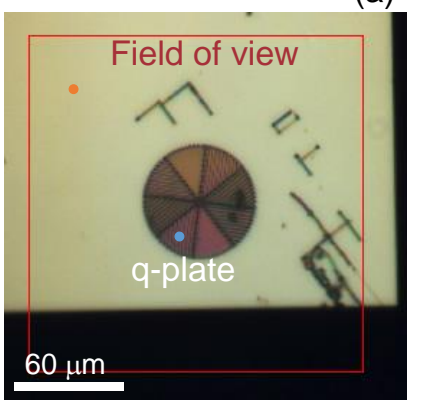

(b)

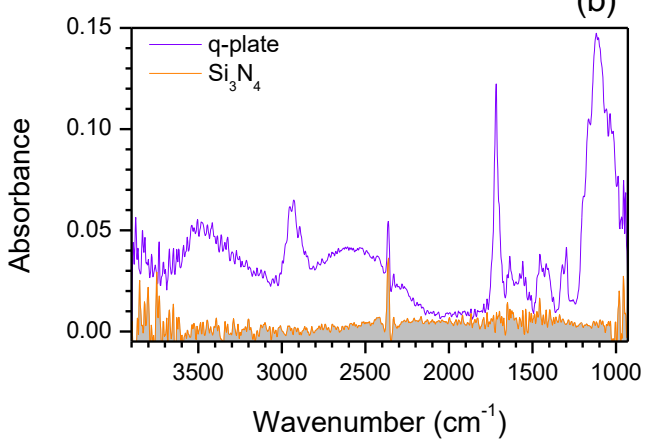

(c)

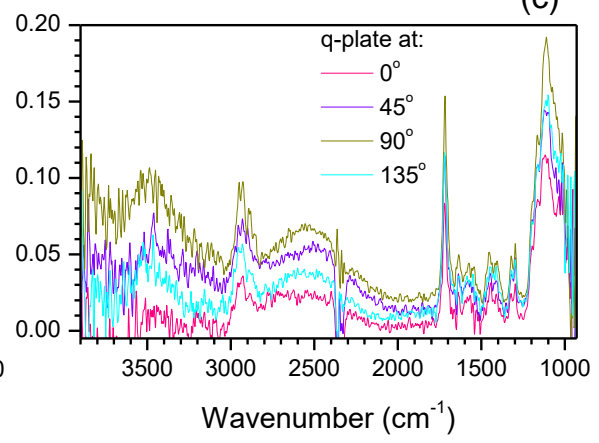

Figure 3. Polarisation dependence of point spectrum of a q-plate; light source is thermal-IR. (a) Optical image of a q-plate polymerized out of SZ2080 resist on a $\mathrm{Si}_{3} \mathrm{~N}_{4} 100-\mathrm{nm}$ membrane. Two color markers show location of point spectrum measurement shown in (b). (c) Angular dependence of point spectrum on the q-plate; $\theta=0^{\circ}$ is a horizontal orientation.
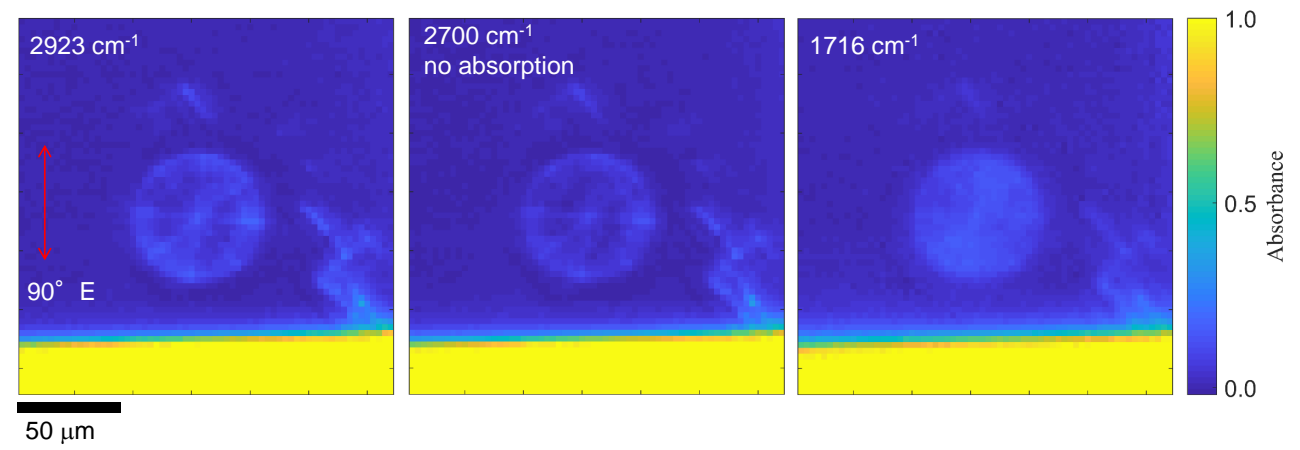

Figure 4. Absorbance images taken using the FPA detector at different wavenumbers for the vertically polarised E-field; light source is thermal-IR.

and peak at $1716 \mathrm{~cm}^{-1}$ due to $\mathrm{C}=\mathrm{O}$ stretching of carbonyl were selected for the orientation mapping together with wavenumber of $2700 \mathrm{~cm}^{-1}$ where there was no specific absorbance in SZ2080. The Q-plate was polymerised using circularly polarised light at $\lambda=800 \mathrm{~nm}$ to avoid polarisation induced effects in the width of the bars, which became larger along direction of polymerisation and depended on direction of laser writing [14].

Figure 4 shows single wavenumber images at one polarisation (vertical $\theta=90^{\circ}$ ) based on the acquired FPA-FTIR image. With measurements at least at four $\theta$ angles, it is possible to recover orientation of the absorbing dipoles, which follows Malus law $\cos ^{2} \theta$ [8]. The resultant orientation maps overlaid with the absorbance are shown in Fig. 5(a). The azimuth plots (Fig. 5(b)) reveal well orientated radial pattern as would be expected for the $q=1$ plate. Since the same alignment maps were observed for the absorption lines $\mathrm{C}=\mathrm{O}$ and $\mathrm{C}-\mathrm{H}$ as well as for the non-absorbing band at $2700 \mathrm{~cm}^{-1}$, the determined same alignment is due to the structure of the q-plate rather than molecular alignment within the polymer. Pixel dimensions $\left(2.8 \times 2.8 \mu \mathrm{m}^{2}\right)$ are slightly larger than the period $\Lambda=2 \mu \mathrm{m}$ of the polymerised pattern. The diffraction limit at $2700 \mathrm{~cm}^{-1}(\lambda=3.7 \mu \mathrm{m})$ was $0.61 \lambda / N A=5.6 \mu \mathrm{m}$ for the used $N A=0.4$. The data shown in Fig. 5 corresponds to the case when resolution is lower than an image discretisation provided by the pixel array of FPA detector. 
(a)

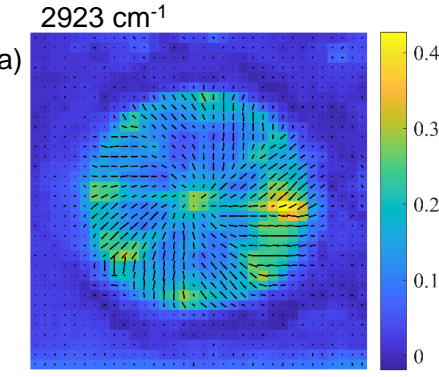

(b)

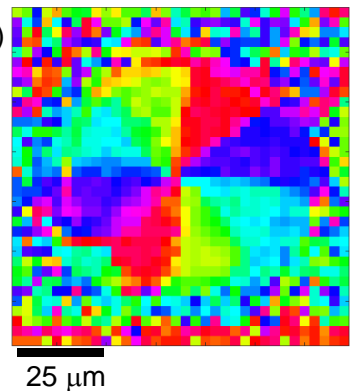

$2700 \mathrm{~cm}^{-1}$
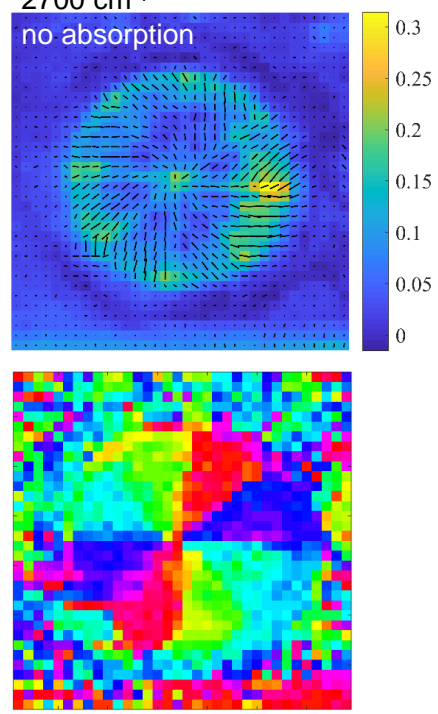
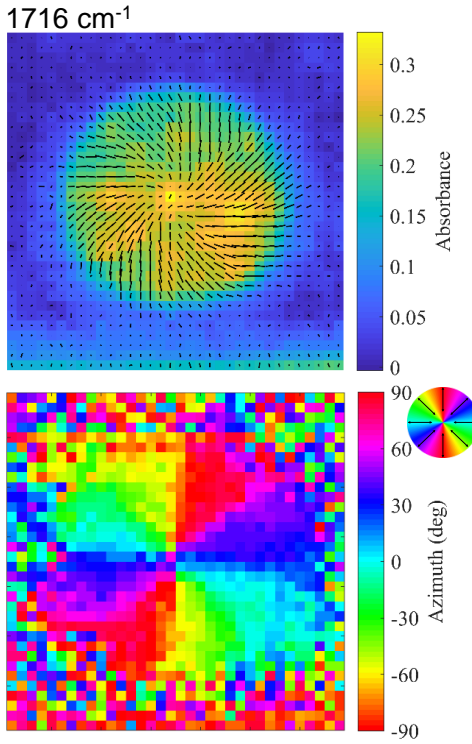

Figure 5. (a) Orientation images at different wavenumbers: at the absorption bands and at the wavenumber of $2700 \mathrm{~cm}^{-1}$ where there were no absorption peak. The orientation azimuth angle $\theta^{\prime}$ was measured from the fit taken at four $\theta$ angles and the length of the black line represents the orientation strength defined by amplitude of min-max absorbance $A_{\max }-A_{\min }$ [7]. (b) Orientation azimuth at the three wavenumbers. Orientation azimuth direction is the same as polymer structure direction.

\subsection{Brown silk}

Brown silk produced by wild moth (Antheraea pernyi) was investigated after standard degumming procedure, which provided single strands [15]. Chemical images of microtomed longitudinal slices of $\sim 10 \mu \mathrm{m}$ thickness embedded in epoxy were acquired using FPA-FTIR microspectroscopy.

Figure 6 shows absorbance and azimuth maps for images obtained by FPA-FTIR technique at four polarisation angles $\theta$ at several absorption bands. The orientation color maps indicate that Amide A $(\mathrm{N}-\mathrm{H})$ and Amide I $(\mathrm{C}=\mathrm{O})$ are mutually aligned along the silk fiber and are perpendicular to the Amide II (C-N) bands. This is an expected result [16] and the flat longitudinal slice was essential to reveal orientation of the absorbing species. The alignment of the molecular absorbers, in this case, is delivered by the secondary $\beta$-sheet structure which has periodicity of $\sim 10 \mathrm{~nm}$ recognisable in $X$-ray diffraction (XRD) patterns of silk. The edges of silk fiber are not well resolved due to the diffraction limit of the used optics ( $N A=0.4$ ) being larger than pixel size of FPA; hence, a blur of silk-epoxy edge occurred. The epoxy region show low absorbance and random orientation as expected from an amorphous host.

The same sample was imaged with FPA-FTIR microspectroscopic technique using the synchrotron IR light source, following the same four $\theta$ angle acquisition procedure and calculation of orientation azimuth. The results (Fig. 7) showed reliable orientation revealed at the focal region. The longer cross section of a rectangular focus [2] had comparable dimensions with the width of the silk fiber slice. The regions out of focus have high noise. However, the absorbance and orientation can be detected much faster with the FPA detector as compared with a single-point mapping measurement usually employed for scanning over the entire area of interest [17]. Therefore, the demonstrated imaging approach is of particular importance for detection of changes in the sample and can potentially be carried out in real time. A simple estimate of acquisition time savings using $64 \times 64 \mathrm{FPA}$ imaging (parallel) compared with single-pixel mapping (sequential) is 4096 times for single image. This is a 

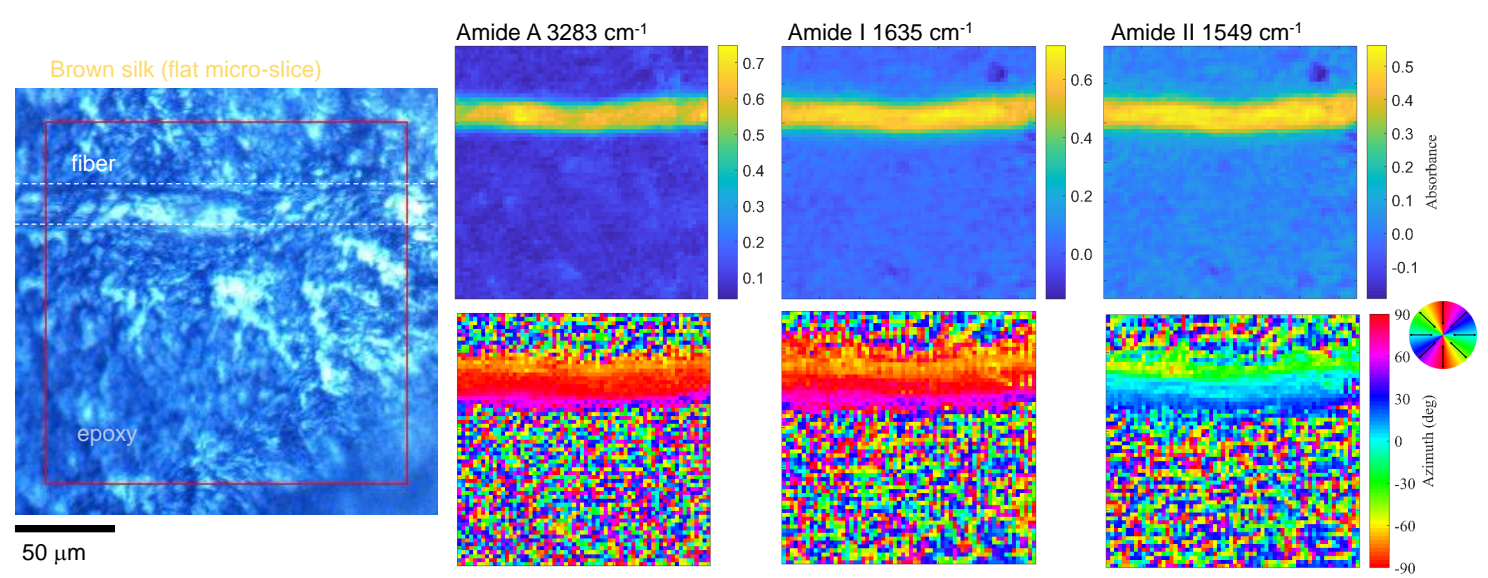

Figure 6. Optical image of a micro-thin brown silk microtome slice and its absorbance and azimuth maps at a single wavelengths of specific bands; the baselines were integrated from 3700 to $3710 \mathrm{~cm}^{-1}$ and $2000-2050 \mathrm{~cm}^{-1}$, for the Amide A and Amide I, II bands, respectively. The light source was thermal-IR.

very large improvement and speeding up of data collection. Considering that four angle measurement was required, an improvement by $10^{3}$ in data collection was gained.

\section{Discussion}

The demonstrated FPA image acquisition in the hyperspectral mode is expected to become a powerful research tool. Even without tight focusing $(N A=0.4)$, it was possible to determine the orientation of the transmitted/absorbed light which originates from sub-wavelength features in $q=1$ plates: a circular metal grating with period of $\Lambda=0.2 \mu \mathrm{m}$ (Fig. 2) and polymerised structures with period $\Lambda=2 \mu \mathrm{m}$ (Fig. 5). Natural silk fibers with secondary protein structures such as $\beta$-sheets with structure length $\sim 10 \mathrm{~nm}$ [18] was revealed using the four probe mode imaging of the absorbance (Fig. 6). This is an interesting feature of this imaging method when the feature size is not spatially resolved but the orientation is determined.

Polarisation composition of synchrotron IR-THz beams is complex, with linear and circular counterparts due to emission occurring at different locations at the edge and inside of a bending magnet [1]. The IR beam is extracted using a mirror with a central slot since synchrotron radiation has a dispersion which is wavelength dependent. X-rays and UV light are transmitted through the central slot, while visible and IR are reflected off the mirror surface, with longer wavelengths at a larger distance from the center. Focusing of IR synchrotron radiation, which is naturally highly focused, into a wider illuminated area suitable for use with the FPA detector, is a formidable engineering challenge [2]. The spectral and polarisation composition of focal spot of tens-of- $\mu \mathrm{m}$ in cross section is not trivial. This study shows, that even without exact knowledge of polarisation in the beam, using measurements with a linear polariser at four $\theta$ orientation angles it was possible to confirm the orientation of absorbers in silk (Figs. 6 vs 7). Non-sliced brown silk fibers were measured with thermal-IR source at the absorbance bands as well as at $2000 \mathrm{~cm}^{-1}$ where no specific absorption peak is present (Fig. A1). Due to circular cross section of silk fibers, the thickness varied at different lateral location on the image. This reduced the possibility to determine the molecular alignment as compared with flat micro-slices of silk (Fig. 6). Also it was revealed that aligned silk fibers were acting as polariser (Figs. A1 and A2).

Another insight, which follows from this application of FPA in FTIR hyperspectral imaging is the possibility to carry out simultaneous four angle measurement when absorption anisotropy of the sample is known. The nano-gratings milled into metallic surface (Fig. 1) are acting as spectrally 


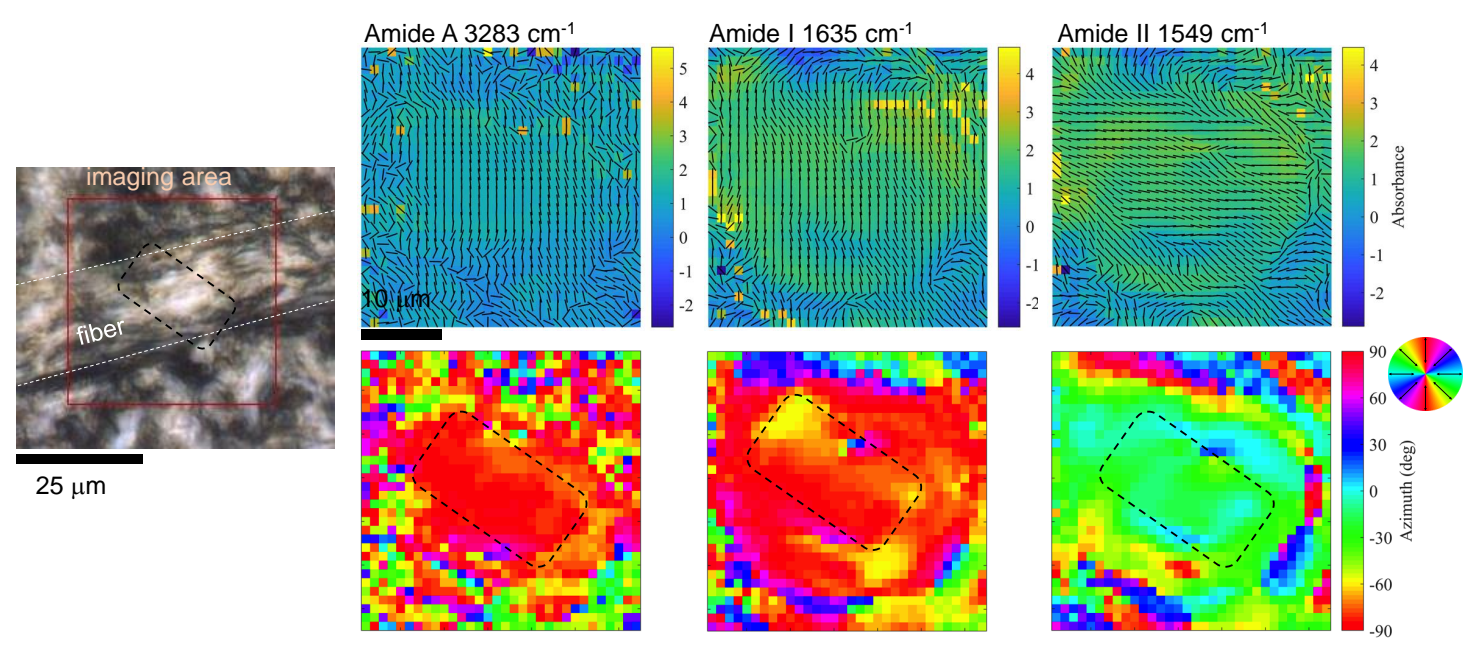

Figure 7. Characterisation of brown silk micro-slice (flat-longitudinal) with synchrotron IR beam. Optical image of a flat longitudinal micro-slice of brown silk fiber in epoxy matrix and maps of absorbance and azimuth. The black dotted rectangle marks the focal region of focused synchrotron radiation [2].

anisotropic wire-grid polarisers and can be imaged over the FPA field of view. This provides a possibility of obtaining four-angle measurement in a single exposure with gratings milled side-by-side at four angles (Fig. A3). Certainly, homogeneity of sample over the region of measurement should be known for unambiguous data interpretation.

Provided a $\lambda$ /4-waveplate at specific IR wavelength is available, a set of four gratings (Fig. A3) can be used to determine Stokes parameters $S=(I, Q, U, V) \equiv\left(S_{0}, S_{1}, S_{2}, S_{3}\right)$ of light, where the total intensity $I=\left|E_{x}\right|^{2}+\left|E_{y}\right|^{2}$ (also a $S_{0}$ parameter), $Q=\left|E_{x}\right|^{2}-\left|E_{y}\right|^{2}\left(S_{1}\right), U=\left|E_{a}\right|^{2}-\left|E_{b}\right|^{2}\left(S_{2}\right)$ where a Cartesian basis $(x, y)$ is rotated by $45^{\circ}$ to obtain $(a, b)$ and $V=\left|E_{l}\right|^{2}-\left|E_{r}\right|^{2}\left(S_{3}\right)$, where SAM $\sigma= \pm 1$ corresponds to the left $(+1)$ and right $(-1)$ polarisations, respectively. The milled metal sub-wavelength gratings provides a capability of polarisation characterisation which is still missing over a wide IR-THz spectral range.

Figure 1 shows the Malus $\cos ^{2} \theta$ pattern along circular grooves, which provides a possibility to determine local orientation azimuth with a higher (at larger radial locations) and lower (at the center) fidelity. A single circular grating can perform as four $45^{\circ}$ rotated linear gratings at quarter segments and, in a single measurement, can provide orientation azimuth. The azimuth determined this way will be a spatial average. The spatial resolution is the highest at the center of concentric grating (lowest resolution of the azimuth) and vice versa, the lowest spatial resolution can be achieved at the farthest radial locations of the grating while the azimuth will be determined there with the highest fidelity. By combining the size of the circular grating and optical magnification of the optical setup, in theory, it is possible to realise four-pixel four-angle detection. This is planned for future work.

\section{Materials and the Method}

FPA-FTIR measurement using a thermal IR source: Offline FPA-FTIR experiment was performed using a Bruker Hyperion 3000 FTIR microscope (Bruker Optik GmbH, Ettlingen, Germany), equipped with a liquid- $\mathrm{N}_{2}$ cooled $64 \times 64$ element FPA detector and a matching $15^{\times}$objective and condenser $(N A=0.40)$, coupled to a Vertex 70 FTIR spectrometer (Bruker Optik GmbH, Ettlingen, Germany) that was equipped with a thermal (Globar(TM)) IR source [2]. FPA-FTIR images were acquired in transmission mode within a $4000-800 \mathrm{~cm}^{1}$ spectral region as a single FTIR image covering 
a sampling area of $182 \times 182 \mu \mathrm{m}^{2}$. Each FTIR spectral image comprised a $64 \times 64$ array of spectra resulting from each square of the detectors on the $64 \times 64$ element FPA array. As a consequence, a single spectrum in each FTIR image represented molecular information acquired from ca. $5.3 \times 5.3 \mu \mathrm{m}^{2}$ area on the sample plane. For each image, high-quality FTIR spectral images were collected at $8 \mathrm{~cm}^{1}$ resolution, with 64 or 128 co-added scans, Blackman-Harris 3-Term apodization, Power-Spectrum phase correction, and a zero-filling factor of 2 using OPUS 7.2 imaging software (Bruker). Background measurements were taken prior to sample spectral images, by focusing on a clean surface area of sample/substrate without the structure using the same acquisition parameters.

Holographic ZnSe wire-grid polarisers (Edmund) were used to set polarisation at the IR spectral range of $\lambda=4000-750 \mathrm{~cm}^{-1}(2.5-13.3 \mu \mathrm{m})$; the extinction of polarisers was $T^{\max } / T^{\min } \simeq 150$ and transmittance $\sim 50 \%$.

FPA-FTIR measurement using synchrotron IR source [2]: Synchrotron-based hyperspectral imaging measurement was performed on the IR Microspectroscopy (IRM) Beamline at the Australian Synchrotron (Victoria, Australia), using a similar Hyperion 3000 microscope and a Vertex 70v FTIR spectrometer system (Bruker Optik GmbH, Ettlingen, Germany). With this setup, the synchrotron FPA-FTIR images were collected with a matching $36^{\times}$objective and condenser $(N A=0.50$; Bruker Optik $\mathrm{GmbH}$, Ettlingen, Germany). Due to the restricted focus size of the synchrotron beam, the FPA detector readout was restricted to $32 \times 32$ pixels, to ensure that spectra were collected only from the most evenly illuminated portion of the detector. The detector readout rates used were $5 \mathrm{kHz}$ for the FPA (actual integration time used was $0.2146 \mathrm{~ms}$ ) with a gain of 3 . The FPA detector had a long wavelength cut-off of $850 \mathrm{~cm}^{-1}$. Using a $36^{\times}$objective, the detector pixel pitch was estimated to be $1.11 \mu \mathrm{m} \times 1.11 \mu \mathrm{m}$ in the sample focal plane.

The absorbance or optical density $A=-\log (T)$ spectrum is defined by the absorption coefficient $\alpha \equiv 4 \pi \kappa / \lambda=2 \omega \kappa / c\left[\mathrm{~cm}^{-1}\right]$ for the transmitted light intensity $I_{T}=I_{0} e^{-\alpha d}=I_{0} \times 10^{-O D}$; where $d$ is thickness of sample, transmittance $T=I_{T} / I_{0}, O D$ is optical density, $\omega$ is the cyclic frequency of light, and $c$ is speed.

\section{Conclusions and Outlook}

FPA-FTIR was used with the four polarisations method to obtain the orientational dependence of the absorbance in three samples with sub-wavelength features. A circular nano-grating was imaged at wavelengths which are by an order of magnitude larger than the diffraction limit, however, the orientational information in the image was revealed. The orientation of absorbing dipoles (silk) or orientation of the structural pattern which is not spatially resolved (q-plates), or polarisation of the transmitted light (circular nano-grating) was revealed in the azimuthal orientation images obtained at four orientations of the linearly polarised light. The pixel dimensions were smaller than the diffraction limit in all cases. Well defined radial orientation of the grooves was recovered by the applied four-angle method. We discuss application of such grating as a polariser for the four-angle method. In laser polymerised, azimuthally orientated gratings - the q-plates - the radial pattern was also reliably retrieved in the orientation images. The highest spatial frequency of molecular absorbers was in brown silk and the orientational azimuth was reliably determined. In addition to the uniform illumination using thermal-IR source we also used focused synchrotron-IR irradiation at higher magnification and the same orientation of the absorption bands in silk was confirmed.

It is shown that the orientation of dipoles, which are sub- $1 \mathrm{~nm}$ objects and are absorbing in the IR spectral window, is determined by the secondary structure of $\beta$-sheets in silk $(\sim 10 \mathrm{~nm})$ or by polymerised rods in q-plates $(\sim 1 \mu \mathrm{m})$. This could have implications in surface-enhanced IR absorption spectroscopy (SEIRAS) for the design of patterns on which a higher sensitivity of detection can be achieved in the IR-THz spectral range [19].

Author Contributions: Conceptualization: J.M. and S.J; methodology: J.V., M.J.T., D.A.; experiments: R.H., M.M., SH.N.; data analysis: R.H. and M.R.; writing-original draft preparation: S.J. and J.M.; writing一review and editing: all authors; sample preparation and fabrication: A.B., V.M., J.-L.L. 
Funding: This research was partially funded by JSPS KAKENHI Grants No.16K06768 and 18H04506. Experiments were carried at the IRM beamline at the Australian Synchroton, part of ANSTO, via the beamtime allocation M13416 and proposal M11615 on THz - Far Infrared beamline for the silk polariser. SJ and VM are grateful for partial support via the "Science for Peace and Security" project NATO SPS 985048.

Conflicts of Interest: The authors declare no conflict of interest.

\section{References}

1. Ryu, M.; Linklater, D.; Hart, W.; Balčytis, A.; Skliutas, E.; Malinauskas, M.; Appadoo, D.; Tan, Y.; Ivanova, E.P.; Morikawa, J.; Juodkazis, S. 3D Printed Polarising Grids for IR-THz Synchrotron Radiation. J. Opt. 2018, 20, 035101.

2. Tobin, M.; Vongsvivut, J.; Martin, D.; Sizeland, K.; Hackett, M.; Takechi, R.; Fimorgnari, N.; Lam, V.; Mamo, J.; Carter, E.; Swarbrick, B.; Layc, P.; Christensen, D.; Perez-Guaita, D.; Lowery, E.; Heraud, P.; Wood, B.; Puskar, L.; Bambery, K. Focal plane array IR imaging at the Australian Synchrotron. Infrared Phys. Technol. 2018, 94, 85 - 90 .

3. Tao, H.; Kaplan, D.L.; Omenetto, F.G. Silk Materials: A Road to Sustainable High Technology. Adv. Mater. 2012, 24, 2824 - 2837.

4. Shao, Z.; Vollrath, F. Surprising strength of silkworm silk. Nature 2002, 418, 741.

5. Rousseau, M.E.; Lefevre, T.; Beaulieu, L.; Asakura, T.; Pezolet, M. Study of Protein Conformation and Orientation in Silkworm and Spider Silk Fibers Using Raman Microspectroscopy. Biomacromolecules 2004, 5, 2247 - 2257.

6. Du, N.; Liu, X.Y.; Narayanan, J.; Li, L.; Lim, M.L.M.; Li, D. Design of Superior Spider Silk: From Nanostructure to Mechanical Properties. Biophysical J. 2006, 91, 4528 - 4535.

7. Honda, R.; Ryu, M.; Balčytis, A.; Vongsvivut, J.; Tobin, M.J.; Juodkazis, S.; Morikawa, J. Paracetamol micro-structure analysis by optical mapping. Appl. Surf. Sci. 2019, 473, 127-132.

8. Hikima, Y.; Morikawa, J.; Hashimoto, T. FT-IR Image Processing Algorithms for In-Plane Orientation Function and Azimuth Angle of Uniaxially Drawn Polyethylene Composite Film. Macromolecules 2011, $44,3950-3957$.

9. Wang, X.; Kuchmizhak, A.A.; Brasselet, E.; Juodkazis, S. Dielectric geometric phase optical elements fabricated by femtosecond direct laser writing in photoresists. Appl. Phys. Lett. 2017, 110, 181101.

10. Marrucci, L.; Karimi, E.; Slussarenko, S.; Piccirillo, B.; Santamato, E.; EleonoraNagali.; Sciarrino, F. Spin-to-orbital conversion of the angular momentum of light and its classical and quantum applications. J. Opt. 2011, 13, 064001.

11. Slussarenko, S.; Murauski, A.; Du, T.; Chigrinov, V.; Marrucci, L.; Santamato, E. Tunable liquid crystal q-plates with arbitrary topological charge. Opt. Express 2011, 19, 4085-4090. doi:10.1364/OE.19.004085.

12. Biener, G.; Niv, A.; Kleiner, V.; Hasman, E. Formation of helical beams by use of Pancharatnam - Berry phase optical elements. Opt. Lett. 2002, 27, $1875-1877$.

13. Hakobyan, D.; Brasselet, E. Left-handed optical radiation torque. Nature Photonics 2014, 82, $610-614$.

14. Rekštytė, S.; Jonavicius, T.; Gailevičius, D.; Malinauskas, M.; Mizeikis, V.; Gamaly, E.G.; Juodkazis, S. Nanoscale precision of 3D polymerisation via polarisation control. Adv. Opt. Mat. 2016, 4, 1209 - 1214.

15. Balčytis, A.; Ryu, M.; Wang, X.; Novelli, F.; Seniutinas, G.; Du, S.; Wang, X.; Li, J.; Davis, J.; Appadoo, D.; Morikawa, J.; Juodkazis, S. Silk: Optical Properties over 12.6 Octaves THz-IR-Visible-UV Range. Materials 2017, 10, 356.

16. Ryu, M.; Bačytis, A.; Wang, X.; Vongsvivut, J.; Hikima, Y.; Li, J.; Tobin, M.J.; Juodkazis, S.; Morikawa, J. Orientational Mapping Augmented Sub-Wavelength Hyper-Spectral Imaging of Silk. Sci. Reports 2017, 7, 7419 .

17. Ryu, M.; Kobayashi, H.; Balčytis, A.; Wang, X.; Vongsvivut, J.; Li, J.; Urayama, N.; Mizeikis, V.; Tobin, M.; Juodkazis, S.; Morikawa, J. Nanoscale chemical mapping of laser-solubilized silk. Mat. Res. Express 2017, 4, 115028.

18. Ryu, M.; Honda, R.; Cernescu, A.; Vailionis, A.; Balcytis, A.; Vongsvivut, J.; Li, J.L.; Linklater, D.P.; Ivanova, E.P.; Mizeikis, V.; Tobin, M.J.; Morikawa, J.; Juodkazis, S. Nanoscale optical and structural characterisation of silk. arXiv 2019, p. arXiv:1902.02688. 
10 of 12

19. Zhizhchenko, A.; Kuchmizhak, A.; Vitrik, O.; Kulchin, Y.; Juodkazis, S. On-demand concentration of an analyte on laser-printed polytetrafluoroethylene. Nanoscale 2018, 10, $21414-21424$. 


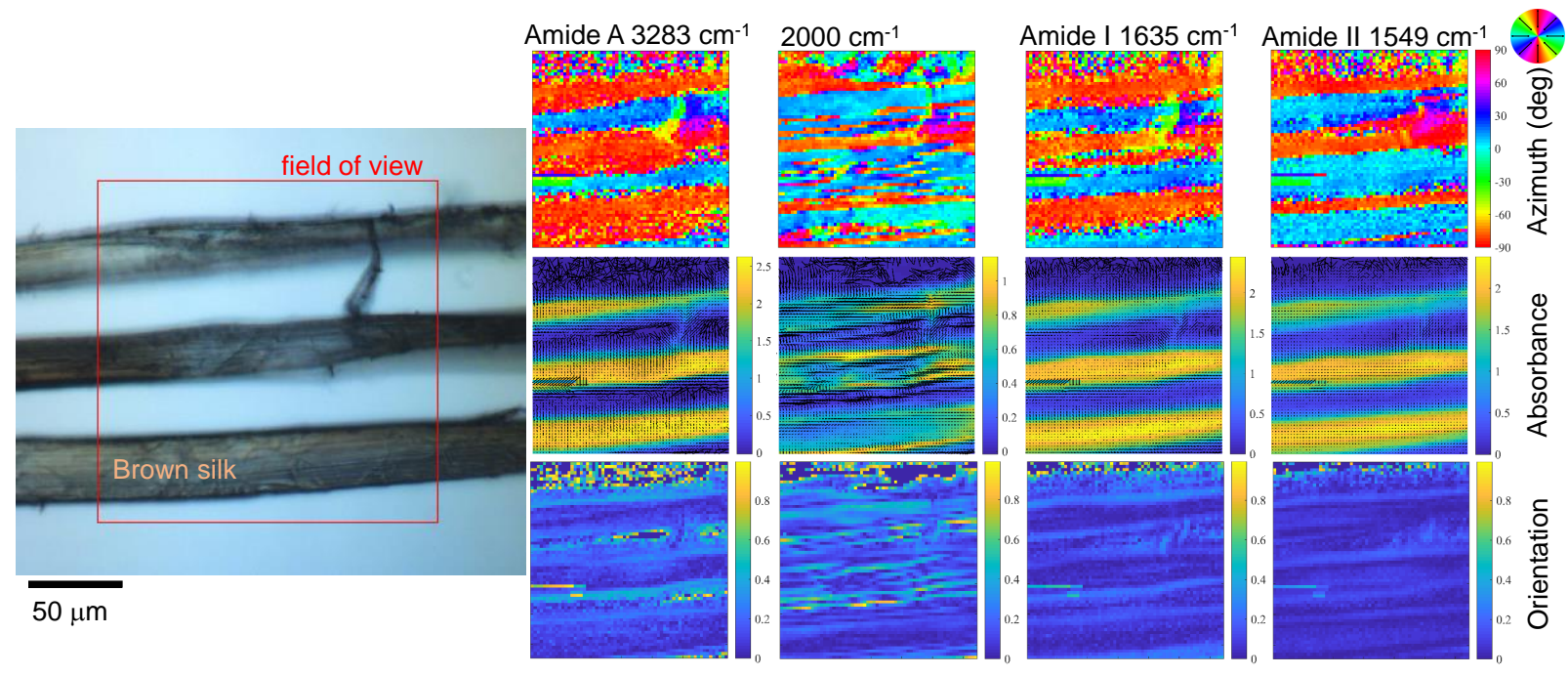

Figure A1. Optical image of brown silk fibers and maps of azimuth, absorbance, and orientation function. Light source was thermal-IR.

\section{Appendix A Supplement}

Figure A1 shows spectral analysis from degummed brown silk fibers at several spectral locations measured with thermal IR source and FPA. The fibers were not sliced. The orientation function used to evaluate ordering of the absorbing dipoles is directly obtained from:

$$
f_{\psi}=\frac{D-1}{D+2} \times \frac{2}{3\left\langle\cos ^{2} \alpha\right\rangle-1}
$$

where the dichroic ratio $D \equiv A_{\max } / A_{\min } \equiv A_{\|} / A_{\perp}$ with $\|$ orientation corresponding to the $\alpha=0^{\circ}$ angle between the molecular chain axis and transition dipole moment, $\langle\ldots\rangle$ is the ensemble average; the orientation of the $\mathrm{CH}_{2}$ dipole moment is defined here as the molecular chain axis. The range of $f_{\psi}$ values is from 0 to 1 (see orientation panels in Fig. A1), where 1 corresponds to the perfect alignment of the molecular chain axis to the orientation direction, and 0 corresponds to the random phase.

The extinction ratio is defined by the maximum-to-minimum transmission ratio $\sigma_{e x}=T_{0} / T_{\pi / 2}$, where $T_{0 ; \pi / 2}$ are the maximum and minimum transmittance values at the two perpendicular orientation angles $=0, \pi / 2$, respectively (Fig. A2). The $\sigma_{e x}$ was measured for the silk grating via a wide far-IR spectral range (Fig. A2). For the $\sigma_{e x}=1$ material or pattern has isotropic transmittance while the metallic grid polariser has $\sigma_{e x} \gg 1$ since the E-field of light polarized along the metallic lines of the grating $\left(\theta=90^{\circ}\right.$; Fig. A2(b)) is reflected (extinct in transmission) while the transmittance $T_{0}$ is maximal. Since $\sigma_{e x}<1$ was not observed, there was no anisotropy induced by a particular molecular alignment in brown silk grating. The anisotropy of absorbance due to molecular alignment can be quantified from the angular dependence of $A(\theta)$ and only four angles with angular separation of $\pi / 4$ are required [8] to retrieve the orientational dependence $A(\theta)=A_{0} \cos ^{2} \theta+A_{\pi / 2} \sin ^{2} \theta$. The maximum $\sigma_{e x} \approx 2.5$ was observed at $210 \pm 10 \mathrm{~cm}^{-1}$ wavenumber. In this range around $48 \mu \mathrm{m}$ wavelength, the aligned silk fibers of comparable diameter were acting as a polariser with $\sigma_{e x}$ larger as compared with 3D printed polymer gratings which do not have particular molecular ordering [1].

Figure A3(a) shows an optical image of the circular gratings of different diameters milled into $100-\mathrm{nm}$-thick Au deposited on 30- $\mu \mathrm{m}$-thick sapphire. The period of gratings is $200 \mathrm{~nm}$ with duty cycle of 0.5 . The orientation of gratings milled by $\mathrm{Ga}^{+}$ions is at four orientation angles which can be used as polarisers for four angle method [8] applied in this study or for polariscopy measurements to determine 

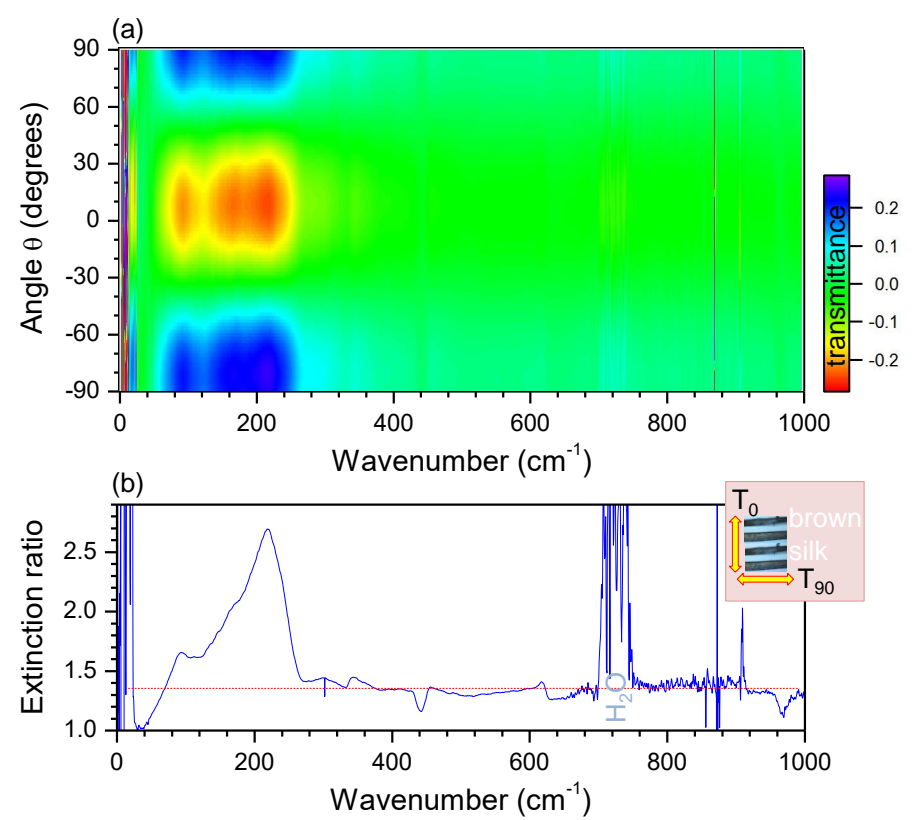

Figure A2. Far-IR measurements of brown silk grating (as shown in the optical image in Fig. A1); radiation source was far-IR synchortron beamline. (a) The orientational transmittance spectrum map at different polarisation angles $\theta$. (b) Extinction ratio (the maximum-to-minimum transmission ratio) for the brown silk grating. The inset shows orientation conventions for the transmittance and extinction ratio $\sigma_{e x}=T_{0} / T_{90}$. Note different wavenumber scales for the panels (a) and (b).

(a)

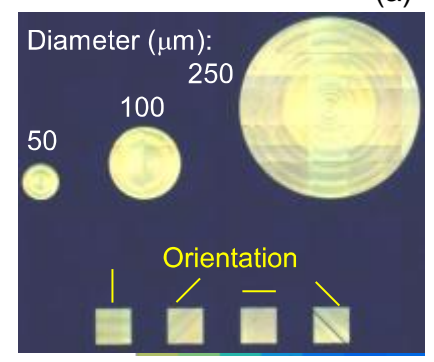

(b)

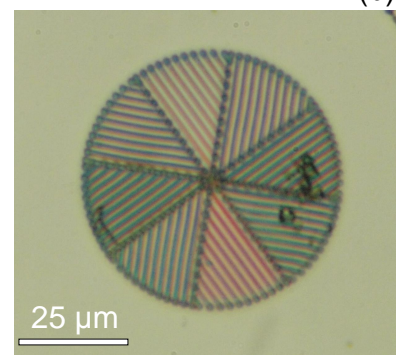

Figure A3. (a) Optical microscope image of circular and 1D gratings milled in 100-nm-thick Au on micro-thin $(30 \mu \mathrm{m})$ sapphire. The smallest circular grating was used in measurement shown in Fig. 1. (b) A closeup optical image of the q-plate used for measurements shown in Fig. 3.

Stokes parameters of an arbitrary polarised light. The panel (b) shows the q-plate polymerised out of SZ2080 resist. 